\title{
Fructus xanthii improves lipid homeostasis in the epididymal adipose tissue of rats fed a high-fat diet
}

\author{
XIUMIN LI ${ }^{1,2}$, MINGXING YANG ${ }^{1}$, ZHIPENG LI ${ }^{1,2}$, MEI XUE $^{1,2}$, ZHAOSHUI SHANGGUAN $^{3}$, \\ ZHIMIN OU ${ }^{1}$, MING LIU ${ }^{1}$, SUHUAN LIU ${ }^{1,3}$, SHUYU YANG ${ }^{1,4}$ and XUEJUN LI ${ }^{1,4}$ \\ ${ }^{1}$ Xiamen Diabetes Institute, The First Affiliated Hospital of Xiamen University, Xiamen, Fujian 361003; \\ ${ }^{2}$ Beijing University of Chinese Medicine, Beijing 100029; ${ }^{3}$ Central Laboratory; ${ }^{4}$ Division of Endocrinology and Diabetes, \\ The First Affiliated Hospital of Xiamen University, Xiamen, Fujian 361003, P.R. China
}

Received January 23, 2015; Accepted November 6, 2015

DOI: $10.3892 / \mathrm{mmr} .2015 .4628$

\begin{abstract}
High fat diet (HFD)-induced obesity triggers common features of human metabolic syndrome in rats. Our previous study showed that Fructus xanthii (FX) attenuates HFD-induced hepatic steatosis. The present study was designed to investigate the effects of FX on lipid metabolism in epididymal fat (EF), and examine its underlying mechanisms. Aqueous extraction fractions of FX or vehicle were orally administered by gavage for 6 weeks to rats fed either a HFD or a normal chow diet (NCD). The levels of circulating free fatty acid (FFA) were determined in plasma, and the expression levels of lipid metabolism- and inflammation-associated genes in the EF were measured using reverse transcription-quantitative polymerase chain reaction analysis. The general morphology, size and number of adipocytes in the $\mathrm{EF}$, and the levels of macrophage infiltration were evaluated using hematoxylin and eosin staining or immunohistochemical staining. FX decreased circulating levels of FFA, increased the expression levels of sterol-regulatory-element-binding protein-1c, FAS, acetyl coenzyme A carboxylase, diacylglycerol acyltransferase and lipoprotein lipase lipogenic genes in the EF. FX increased the numbers of adipocytes in the EF, and featured a shift towards smaller adipocyte size. Compared with the vehicle-treated rats, positive staining of F4/80 was more dispersed in the FX-treated rats, and the percentage of F4/80 positive cells was significantly decreased. FX attenuated HFD-induced lipid dyshomeostasis in the epididymal adipose tissue.
\end{abstract}

Correspondence to: Dr Xuejun Li or Dr Shuyu Yang, Xiamen Diabetes Institute, The First Affiliated Hospital of Xiamen University, 55 Zhenhai Road, Xiamen, Fujian 361003, P.R. China

E-mail: xmlixuejun@163.com

E-mail: xmyangshuyu@126.com

Key words: Fructus xanthii, traditional Chinese medicine, high-fat diet, epididymal fat, lipid metabolism, inflammation

\section{Introduction}

Obesity, specifically the enlargement of visceral adiposity, is considered the major risk factor for the development of insulin resistance, a characteristic feature of diabetes and metabolic syndrome (1), which is often characterized by nonalcoholic fatty liver disease (NAFLD) (2). The metabolic pathways leading to hepatic steatosis include enhanced non-esterified fatty acid released from adipose, increased de novo lipogenesis (DNL), decreased $\beta$-oxidation and reduced very low density lipoprotein (VLDL) export (3).

Adipocytes posses the full complements of enzymes and regulatory factors required to execute DNL and lipolysis, and the two tightly controlled biochemical processes determine the rate of lipid metabolism (4). Sterol-regulatory-element-binding protein-1c (SREBP 1c), a key regulator for lipid metabolism that is involved in adipocyte differentiation, is expressed at high levels in the adipose tissue and stimulates the expression of several lipogenic genes, including FAS, acetyl-CoA carboxylase (ACC), stearyl-CoA desaturase 1 (SCD 1) and lipoprotein lipase (LPL) (4). LPL is the rate-limiting enzyme for the import of triglyceride (TG)-derived fatty acids from VLDLs or chylomicrons for storage by the adipose tissue (5). TG synthesis and storage in the adipose tissue are important in maintaining metabolic homeostasis (6). The first committed step in TG synthesis via the glycerol phosphate pathway is mediated by glycerol-3-phosphate acyltransferase (GPAT) enzymes (7). An additional fatty acid is subsequently transferred to lysophosphatidic acid by the family of 1-acylglycerol-3-phosphate acyltransferase (AGPAT) enzymes to produce phosphatidate, which serves as a precursor of acidic phospholipids or diacylglycerol (DAG) (8). The DAG is converted to TG through the action of diacylglycerol acyltransferase (DGAT) enzymes (9).

The hormonal regulation of lipolysis in adipocytes provides a main switch between lipid storage and lipid mobilization in response to dietary requirements. Hormone-sensitive lipase (HSL) is activated and translocated to the lipid droplet surface, where it interacts with specific lipid droplet proteins, including perilipin and fat-specific protein-27 (FSP-27), which are regulated by peroxisome proliferator-activated receptor (PPAR) $\gamma(10)$. Of note, HSL works in concert with other lipases, including adipose triglyceride lipase (ATGL), 
to maximize the lipolytic output (11). Among the factors that contribute to enhanced lipolysis associated with obesity, tumor necrosis factor (TNF) $\alpha$ and adipocyte size appear to be the most relevant. TNF $\alpha$, secreted from the macrophages and adipocytes within the adipose tissue of obese humans and animals, chronically stimulates lipolysis (12). Monocyte chemoattractant protein (MCP-1) is produced at high levels in obese fat pads and, therefore, attracts a higher number of macrophages (13). Taken together, obesity, particularly the excess accumulation of fat in intra-abdominal depots, causes and/or exacerbates metabolic disorders, independently and in association with other diseases (14). Obesity is associated with an increased risk of hepatic steatosis, and the incidence of steatosis is correlated with the degree of obesity (15).

Fructus xanthii (FX), termed Cang-Erzi in Chinese Pin Yin, was first recorded in Qian Jin dietetic therapy, and is commonly used as a traditional Chinese medicine for treating sinusitis and headache due to rheumatism (16). Data from animal experiments have confirmed that FX possesses antioxidant, antinociceptive and anti-inflammatory properties, and can protect pancreatic $\beta$-cells form cytokine-induced damage (17,18). In our previous study, it was observed that FX attenuates HFD-induced heptic steatosis, suppresses fatty acid $\beta$-oxidation and upregulates the expression levels of inflammatory genes in the liver (19). Steatosis is the result of ectopic lipid accumulation in the liver, and contributes to liver-specific diseases (20). Adipose tissue is the lipid storage organ, thus the dysfunctional lipid storage in adipocytes is a sentinel event in the progression toward metabolic disorder in HFD-induced obesity (21). The forced expansion of adipose tissue prevents metabolic disease, despite gross obesity (22), which supports the hypothesis that lipid 'spill-over' from fat promotes metabolic disease by fostering ectopic lipid deposition (21). The dysfunction of adipose tissue may trigger or exacerbate lipid accumulation in the liver. The present study aimed to investigate the role of FX in the regulation of lipid metabolism and storage in EF in order to determine the role of FX in NAFLD.

\section{Materials and methods}

Preparation of $F X$. The raw material of FX was provided by the Department of Pharmacy of the First Affiliated Hospital of Xiamen University, (Xiamen, China). The herbs were immersed in a $10 \mathrm{X}$ volume of water (V/V) for $30 \mathrm{mins}$, and then decocted twice at boiling temperature for $2 \mathrm{~h}$. The decocted liquids were collected, filtrated, concentrated to $114 \mathrm{mg} / \mathrm{ml}$, and were then placed in a sterile bottle $(30 \mathrm{ml})$ and stored in a refrigerator. Fingerprint analysis of FX was performed using high performance liquid chromatography from three different batches of FX preparation. All chemicals were of analytical grades. Caffeic acid and chlorogenic acid were purchased from Sigma-Aldrich (St. Louis, MO, USA). 3, 4-Dihydroxybenzoic acid, neochlorogenic acid, isochlorogenic acid $\mathrm{C}$, cynarin and 4-dicaffenolyquinic acid were obtained from Sigma-Aldrich.

HPLC separation was performed on C18 column $\left(250 \times 4.6 \mathrm{~mm}^{3} ; 5 \mu \mathrm{m}\right)$. The mobile phase consisted of solvent A (methanol) and solvent B (water containing $0.2 \%$ formic acid). The gradient elution program was as follows: 0-20 min, 2-5\% A; 20-30 min, 5-8\% A; 30-45 min, 8-15\% A; 45-55 min, 15-25\%; 55-60 min, 25\% A; 60-70 min, 25-35\% A; 70-75 min,
35-38\% A; 75-95 $\min , 38-65 \% \mathrm{~A}$; and 95-105 min. A was isocratic at $65 \%$. The UV wavelength was set at $260 \mathrm{~nm}$, column temperature was kept at $25^{\circ} \mathrm{C}$ and the flow rate was set at $1.0 \mathrm{ml} / \mathrm{min}$. Three batches of FX were detected under the same conditions. The results showed the same retention time and similar peak area, indicating that the composition of the FX decoction was consistent and reproducible (19).

Animals and experimental diets. All animal experiments were approved by the Animal Care and Use Committee of Xiamen University. Fifty male Sprague-Dawley rats (Shanghai Experimental Animal center, Chinese Academy of Sciences), (age, 6 weeks; weight, 180-200 g) were housed in standard cages in a temperature $\left(22 \pm 1^{\circ} \mathrm{C}\right)$ and humidity (50-60\%)-controlled room, and maintained in a $12 \mathrm{~h}$ light/dark cycle. All the rats were provided with unrestricted rodent chow and water. Following acclimatization for 1 week, the rats were separately fed a normal chow diet (NCD; control) or a high fat diet (HFD) for 6 weeks. The animals were then subdivided into the following four groups $(n=10)$ : NCD group; HFD group, in which animals received equivalent volume of vehicle; HFD+FX (570 mg/kg/day); and HFD+FX (1,140 mg/kg/day) groups, in which the animals in were treated with FX for another 6 weeks. At the end of the treatment period, the rats were anesthetized with pentobarbital $(40 \mathrm{mg} / \mathrm{kg}$ body weight; Sigma-Aldrich); 5-7 ml blood was collected, and EF samples were removed and stored at $-80^{\circ} \mathrm{C}$ until analysis. Following tissue harvest, the rats were sacrificed by exsanguination while under anesthesia with pentobarbital ( $40 \mathrm{mg} / \mathrm{g}$ body weight). The study was approved by the ethics committee of Xiamen University (Xiamen, China; SYXK 2013-0006).

Free fatty acid (FFA) assay. The blood samples were centrifuged at 3,000 x $\mathrm{g}$ for $5 \mathrm{~min}$, and the plasma was separated and transferred to Eppendorf tubes for storage at $-80^{\circ} \mathrm{C}$ prior to analysis. The concentration of FFA in the plasma was measured using a Free Fatty Acid Quantification kit (Abcam, Cambridge, UK).

RNA extraction and reverse transcription-quantitative polymerase chain reaction (RT-qPCR) analysis. Total RNA was extracted from the subcutaneous fat (SCF) and EF using an RNAsimple Total RNA kit (Tiangen Biotech Co., Ltd., Beijing, China), according to the manufacturer's protocol. Isolated RNA was quantified using a NanoDrop ND-1000 spectrophotometer (NanoDrop Technologies; Thermo Fisher Scientific, Inc., Waltham, MA, USA).

The reverse transcription of total RNA into cDNA was performed using a FastQuanti RT kit with gDNAse (Tiangen Biotech Co., Ltd.), according to the manufacturer's protocol. qPCR was conducted in a total volume of $20 \mu \mathrm{l}(2 \mathrm{X} 10 \mu \mathrm{l}$, SuperReal PreMix, 50X 0.4 $\mu$ l ROX Reference Dye, $2 \mu 1$ DNA template, $6 \mu \mathrm{l}$ RNase-Free $\mathrm{ddH}_{2} \mathrm{O}, 0.8 \mu \mathrm{l}$ forward primer and $0.8 \mu \mathrm{l}$ reverse primer. The reaction was performed as follows: $15 \mathrm{~min}$ at $95^{\circ} \mathrm{C}, 40$ cycles of $95^{\circ} \mathrm{C}$ for $10 \mathrm{sec}, 60^{\circ} \mathrm{C}$ for $20 \mathrm{sec}$, $72^{\circ} \mathrm{C}$ for $20 \mathrm{sec}$ and $15 \mathrm{sec}$ incubation at $95^{\circ} \mathrm{C}$. Gene expression analysis was performed using the comparative quantification cycle $(\mathrm{Cq})$ method (23). Amplification of the $\beta$-actin sequence was performed in parallel and was used to normalize values obtained for target genes. The primers (Shanghai Life 
Table I. Oligonucleotide primers used for reverse transcription-quantitative polymerase chain reaction analysis.

\begin{tabular}{|c|c|c|}
\hline Gene & Forward primer & Reverse primer \\
\hline ChREBP & 5'-CTCGTGCAGGTCATCAAGAA-3' & 5'-CAGCCCTCTTCATCTCCAAG-3' \\
\hline SREBP-1c & 5'-GGAGCCATGGATTGCACATT-3' & 5'-CCTGTCTCACCCCCAGCATA-3' \\
\hline LXR & 5'-TCAGCATCTTCTCTGCAGACCGG-3' & 5'-TCATTAGCATCCGTGGGAACA-3' \\
\hline PPAR- $\alpha$ & 5'-CTCGTGCAGGTCATCAAGAA-3' & 5'-CAGCCCTCTTCATCTCCAAG-3' \\
\hline FAS & 5'-CACAGCATT CAGTCCTATCCACAGA-3' & 5'-CACAGCCAACCAGATGCTTCA-3' \\
\hline ACC1 & 5'-GGACAGACTGATCGCAGAGAAAG-3' & 5'-TGGAGAGCCCCACACACA-3' \\
\hline SCD-1 & 5'-CCTTAACCCTGAGATCCCGTAGA-3' & 5'-AGCCCATAAAAGATTTCTGCAAA-3' \\
\hline CPT-1 & 5'-AATTGCAGTGGTATTTGAAGCTAA AA-3' & 5'-GATATATTCTTCCCACCAGTCACTCA-3' \\
\hline $\mathrm{ACO}$ & 5'-GATTCAAGACAAAGCCGTCCAG-3' & 5'-TCCACCAGAGCAACAGCATTG-3' \\
\hline ACOX1 & 5'-GTTGATCACGCACATCTTGGA-3' & 5'-TCGTTCAGAATCAAGTTCTCAATTTC-3' \\
\hline $\mathrm{C} / \mathrm{EBP}$ & 5'-TTACAACAGGCCAGGTTTCC-3' & 5'-GGCTGGCGACATACAGTACA -3'; \\
\hline $\operatorname{PPAR} \gamma$ & 5'-ATTCTGGCCCACCAACTTCGG-3' & 5'-TGGAAGCCTGATGCTTTATCCCCA-3' \\
\hline GPAT & 5'-TGATCAGCCAGGAGCAGCTG-3' & 5'-AGACAGTATGTGGCACTCTC-3' \\
\hline AGPAT & 5'-GCATTTCAGGATCTCGTTCACA -3' & 5'-ATCAACCCAACGAGAGCACTTT-3' \\
\hline DPAT & 5'-AGACTAGGAGGAGTGTGCAGGC -3' & 5'-CGCTTCTTCCAAGGGAACTATG-3' \\
\hline ATGL & 5'-CACTTTAGCTCCAAGGATGA-3' & 5'-TGGTTCAGTAGGCCATTCCT-3' \\
\hline HSL & 5'-CGCCTTACGGAGTCTATGC-3' & 5'-TCTGATGGCTCTGAGTTGC-3' \\
\hline LPL & 5'-CAGCTGGGCCTAACTTTGAG-3' & 5'-CCTCTCTGCAATCACACGAA-3' \\
\hline CD36 & 5'-CGGCGATGAGAAAGCAGAA-3' & 5'-CAACCAGGCCCAGGAGC-3' \\
\hline perlipin & 5'-AGAGGAGACAGATGAGGAGGAAG-3' & 5'-AGATGGTGTTCTGCAGAGTCTTC-3' \\
\hline FSP27 & 5'-CСТTTCCCAGAAGCCAACT-3' & 5'-AGAGTCCAGCGGAGCATT-3' \\
\hline MCP1 & 5'-AATGGGTCCAGAAGTACATTAGAAA-3' & 5'-GGTGCTGAAGTCCTTAGGGTTG -3' \\
\hline TNF- $\alpha$ & 5'-ACACCATGAGCACGGAAAGC-3' & 5'-CCGCCACGAGCAGGAA-3' \\
\hline IL-1 $\beta$ & 5'-AATGGACAGAACATAAGCCAACA-3' & 5'-CCCAAGGCCACAGGCAT-3' \\
\hline CD68 & 5'-TAGTTCTTTCTCCAGCAATTCACC-3' & 5'-CCCGAAGTGTCCCTTGTCC-3' \\
\hline$\beta$-actin & 5'-CGGTCAGGTCATCACTATCG-3' & 5'-TTCCATACCCAGGAAGGAAG-3 \\
\hline
\end{tabular}

Biotechnology Co., Ltd., Guangzhou, China) used are listed in Table I.

Quantitation of adipocyte size. The EF samples were fixed with 4\% paraformaldehyde (Sigma-Aldrich) and embedded in paraffin (Sigma-Aldrich). Standard $4 \mu \mathrm{m}$ thick sections were stained with hematoxylin and eosin (Sigma-Aldrich). Adipocyte size, which was determined as the average adipocyte area (in $\mu \mathrm{m}^{2}$ ) was measured using Image $\mathrm{J}$ software (National Institutes of Health, Bethesda, MD, USA). Adipocyte sizes were measured from EF samples from 10 rats in each group.

Immunohistochemistry. Tissue sections were deparaffinized with xylene (Sinopharm Chemical Reagent Co., Ltd., Shanghai, China) and stepwise rehydrated with serial dilutions of ethanol. For epitope retrieval, slides were incubated in $0.01 \mathrm{M}$ citric acid buffer (Sinopharm Chemical Reagent Co., Ltd.) at $100^{\circ} \mathrm{C}$ for $2 \mathrm{~min}$. Endogenous horseradish peroxidase activity was quenched by incubation with $3 \%$ hydrogen peroxide Sinopharm Chemical Reagent Co.,Ltd, Shanghai, China for $10 \mathrm{~min}$. The tissue sections were then blocked in goat serum and incubated with monoclonal rabbit anti-rat primary antibody against F4/80 (1:100; cat. no. ab111101; Abcam) diluted in blocking buffer at $4^{\circ} \mathrm{C}$ overnight. Incubation with secondary antibodies was performed using an Ultrasensitive

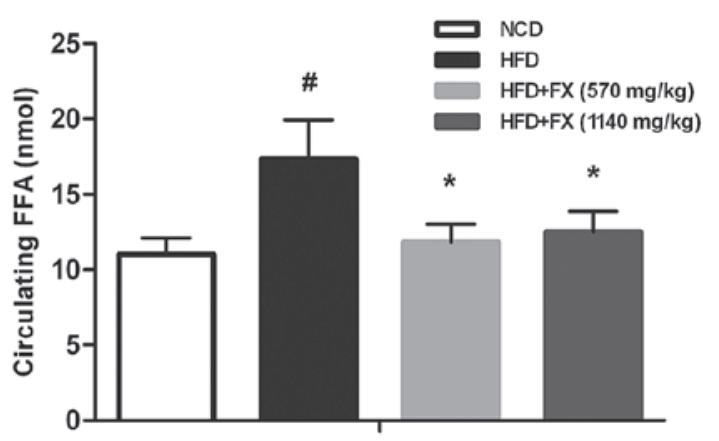

Figure 1. Effect of FX on circulating FFA levels. Data are presented as the mean \pm standard error fo the mean. $(n=10)$. ${ }^{\#} \mathrm{P}<0.05$, vs. NCD group; ${ }^{*} \mathrm{P}<0.05$, vs. HFD group. FX, Fructus xanthii; FFA, free fatty acids; NCD, normal chow diet; HFD, high fat diet.

SP IHC kit (Maixin, Fuzhou, China) and detected using 3, 3'-diaminobenzidine (Solarbio, Beijing, China). Each step was ended by washing in buffer for 5 min twice. All sections were counterstained with hematoxylin prior to dehydration and coverslip placement. From each animal, more than five tissue sections, including representative sections, were analyzed. The images were captured by a Leica microscope (DMI3000B, Leica Microsystems, Inc., Buffalo Grave, IL, USA). 
A

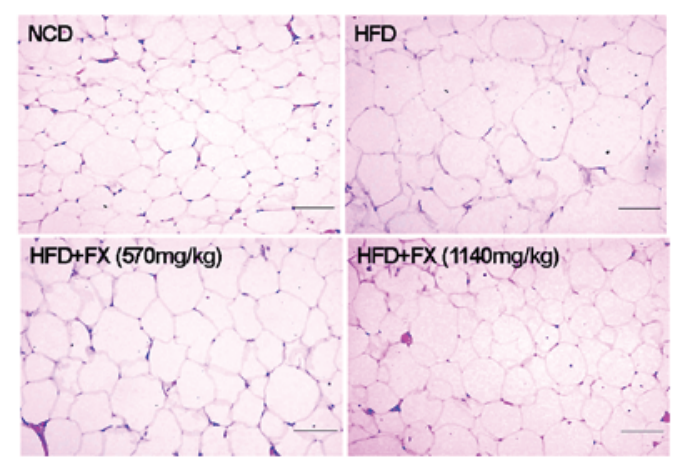

C

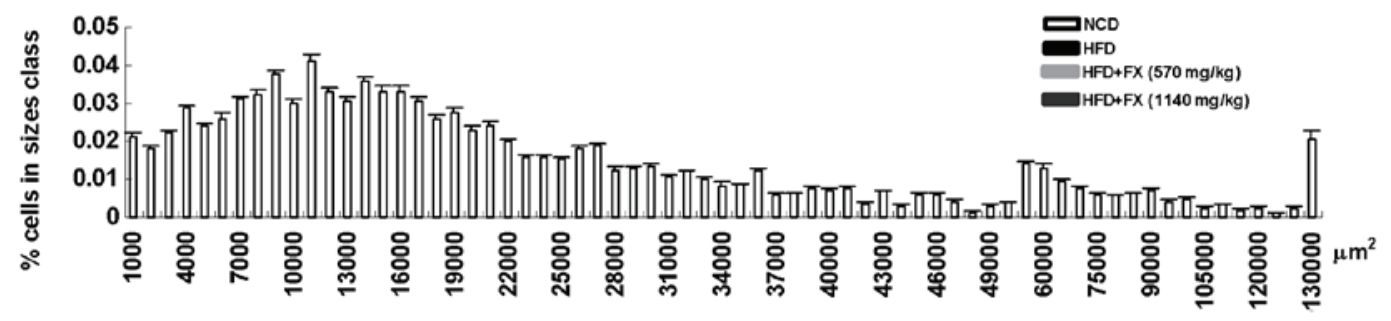

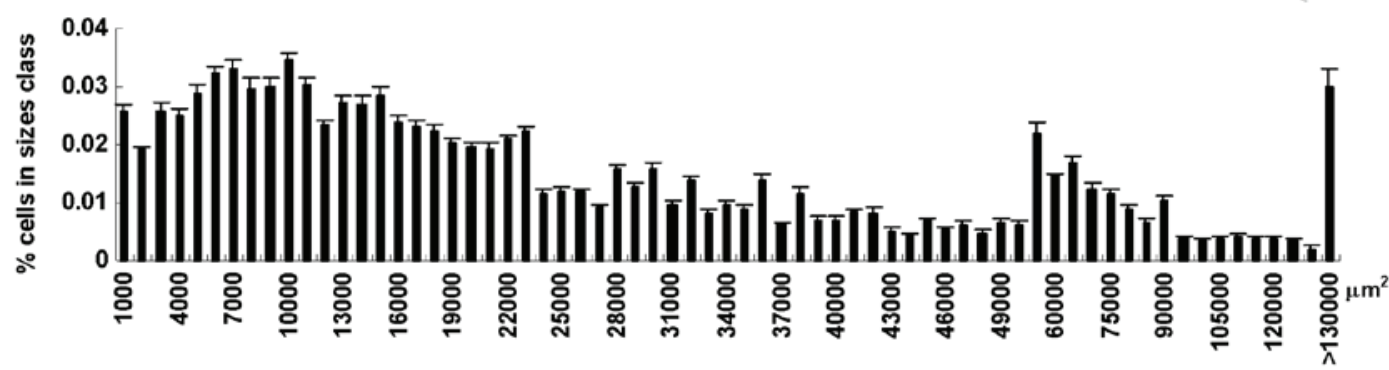

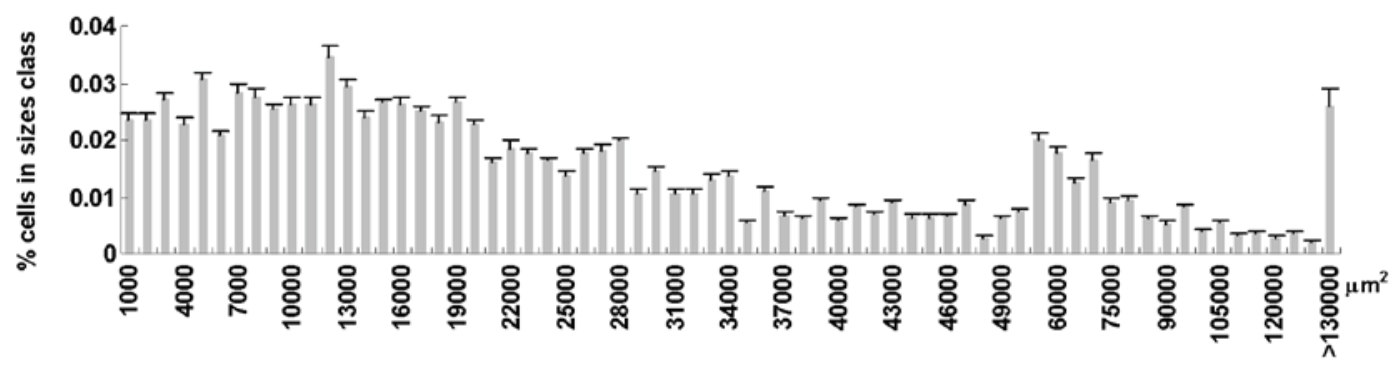

D
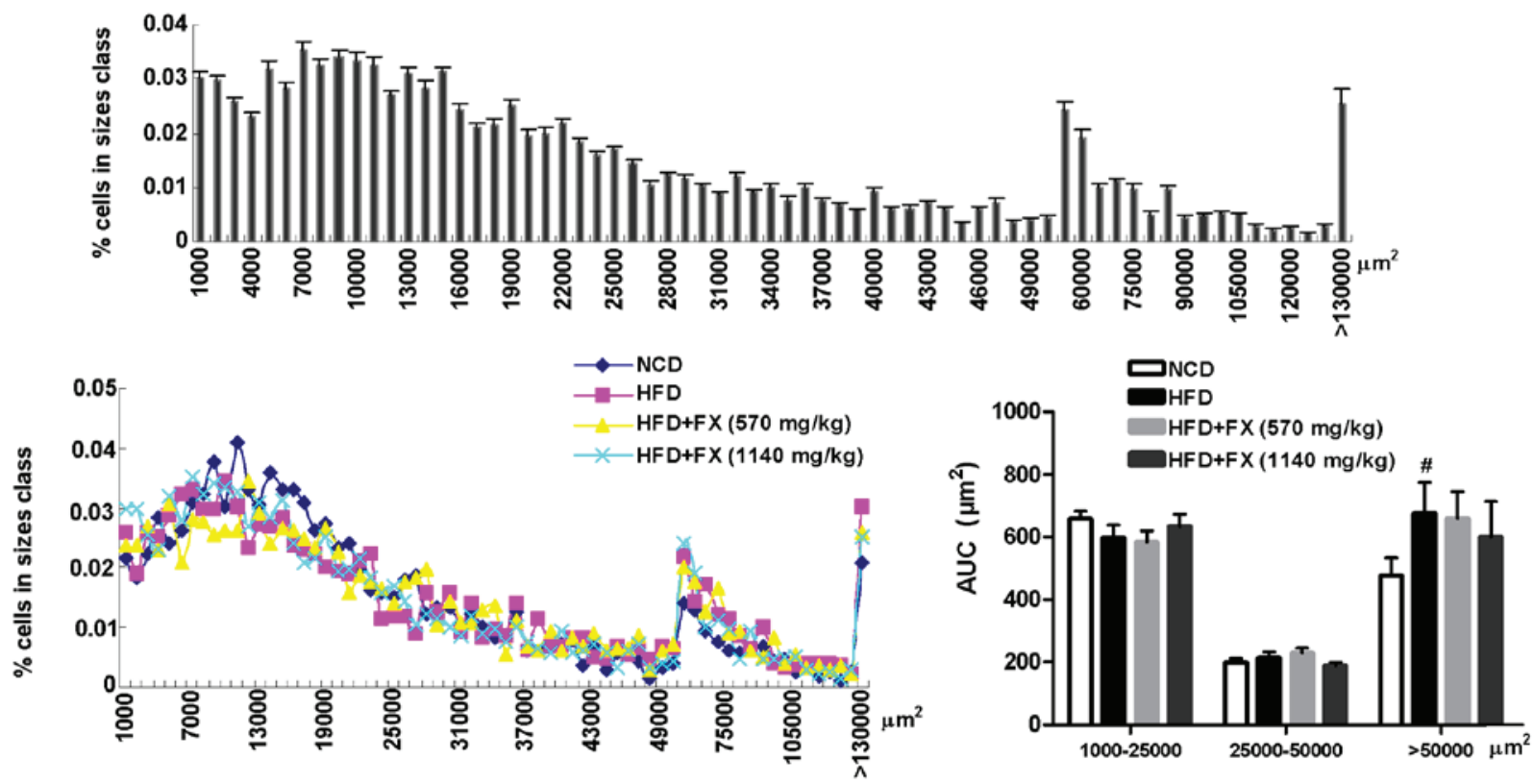

Figure 2. Effect of FX on epididymal adipose tissue mass. (A) Histological analysis of epididymal adipose tissue. Scale bar, $200 \mu \mathrm{m}$ (B) Adipocyte numbers.

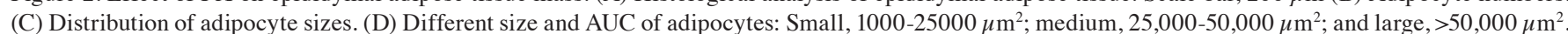
Data are presented as the mean \pm standard error of the mean $(\mathrm{n}=10) .{ }^{*} \mathrm{P}<0.05$, vs. NCD group; ${ }^{*} \mathrm{P}<0.05$, vs. HFD group. FX, Fructus xanthii; NCD, normal chow diet; HFD, high fat diet. AUC, area under the curve. 
$\mathbf{A}$

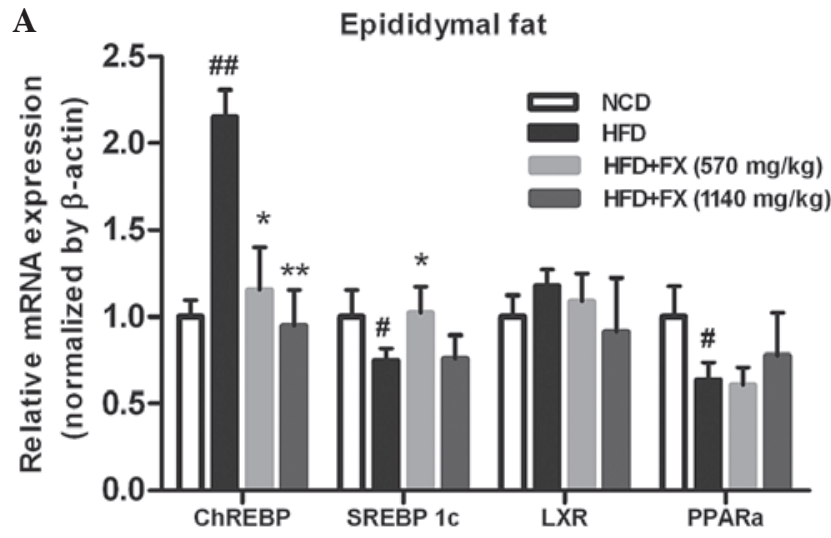

B

Epididymal fat

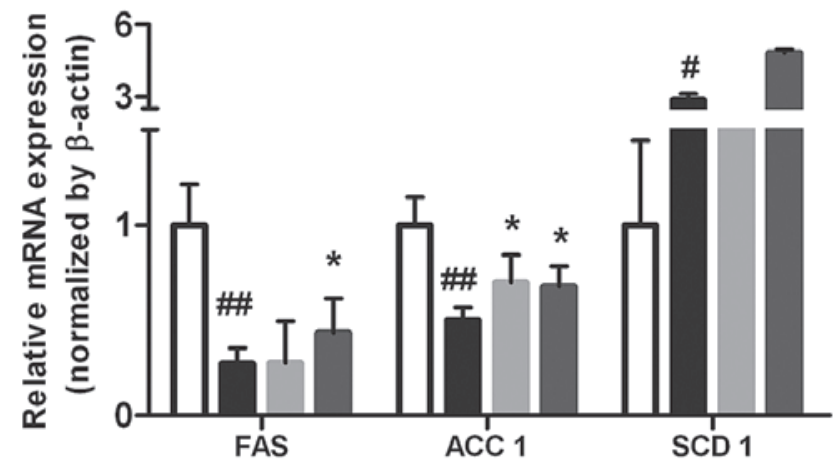

C

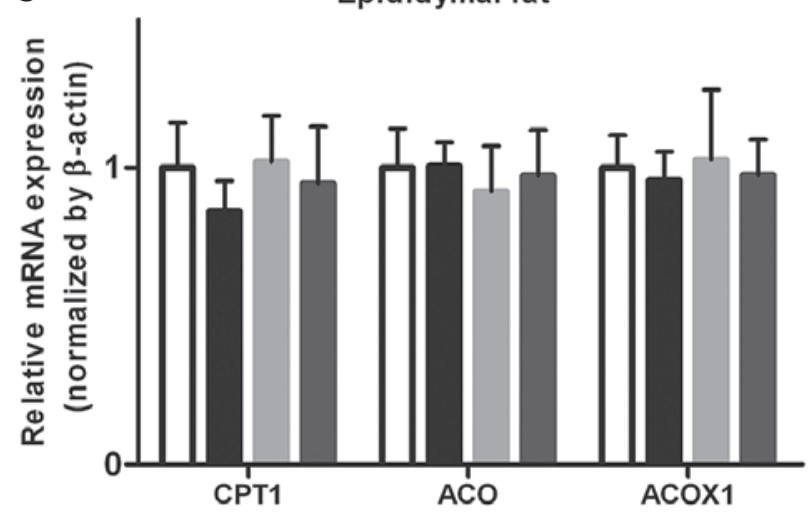

Figure 3. Effect of FX on the expression levels of lipogenic genes in the EF. (A) Expression levels of lipogenic genes; (B) Expression levels of antioxidant genes; (C) Expression levels of lipolytic genes. Data are presented as the mean \pm standard error of the mean $(\mathrm{n}=10)$. ${ }^{\#} \mathrm{P}<0.05$ and ${ }^{\# \#} \mathrm{P}<0.01$, vs. NCD group; ${ }^{*} \mathrm{P}<0.05$ and ${ }^{* *} \mathrm{P}<0.01$, vs. HFD group. FX, Fructus xanthii; NCD, normal chow diet; HFD, high fat diet; ChREBPl, carbohydrate-responsive element-binding protein; SREBP1c, sterol-regulatory-element-binding protein-1c; LXR, liver X receptor; PPAR $\alpha$, peroxisome proliferator-activated receptor $\alpha$; ACC1, acetyl-CoA carboxylase; SCD1, stearyl-CoA desaturase 1; CPT1, carnitine palmitoyltransferase 1; ACP, acyl-acyl carrier protein; ACOX, acyl-CoA oxidase; ACOX1, acyl-CoA oxidase 1.

Statistical analysis. In the present study, all results are presented as the mean \pm standard error of the mean. Statistical analyses were conducted using GraphPad Prism 5 (GraphPad Software Inc., La Jolla, CA, USA) Individual differences between the HFD and FX group were assessed using one-way analysis of variance or Student's t-test, when applicable. $\mathrm{P}<0.05$ was considered to indicate a statistically significant difference.
A

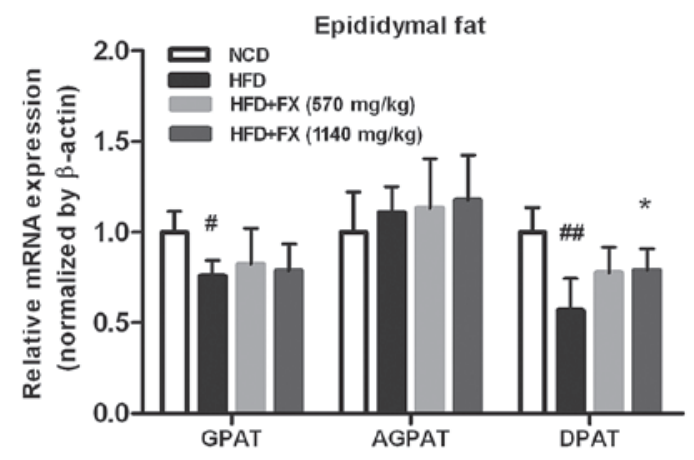

B

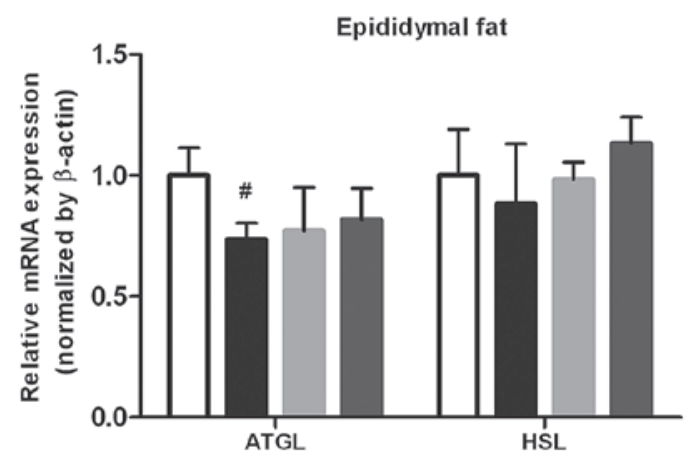

C
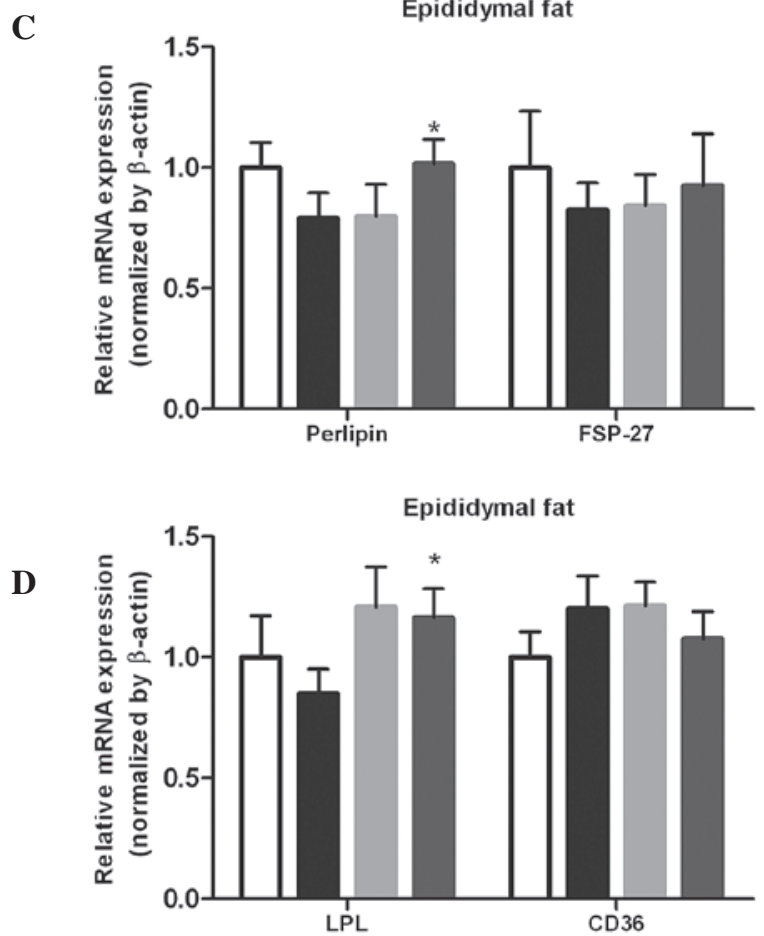

Figure 4. Effect of FX on the expression of lipogenesis-associated genes in the EF. (A) Lipogenesis enzymes; (B) lipolytic enzymes; (C) lipid droplet-associated proteins; (D) genes involved in lipid transport into adipocytes. Data are presented as the mean \pm standard error of the mean $(\mathrm{n}=10) .{ }^{\#} \mathrm{P}<0.05,{ }^{\# \#} \mathrm{P}<0.01$, vs. NCD group; ${ }^{*} \mathrm{P}<0.05$ and ${ }^{* *} \mathrm{P}<0.01$, vs. HFD group. FX, Fructus xanthii; NCD, normal chow diet; HFD, high fat diet. ATGL, adipose triglyceride lipase; HSL, hormone-sensitive lipase; FSP-27; fat specific protein-27; LPL, lipoprotein lipase.

\section{Results}

FX decreases serum levels of FFA. The HFD group were found to have increased serum concentrations of FFA, which 
A

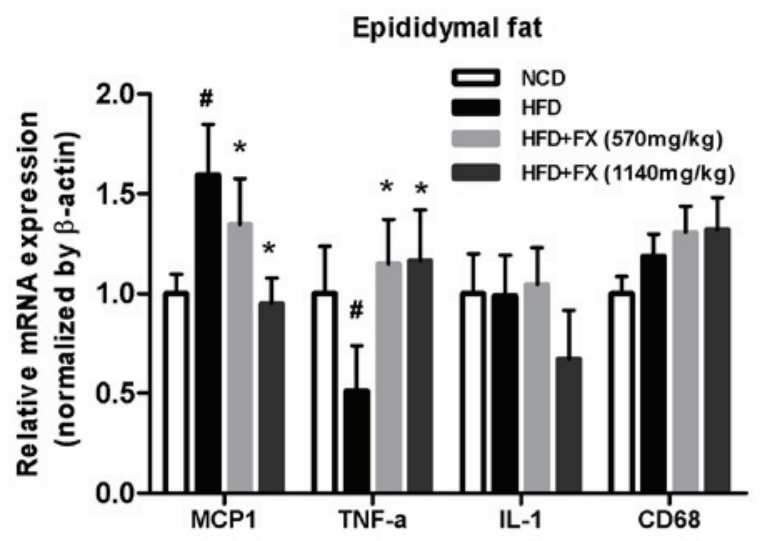

B
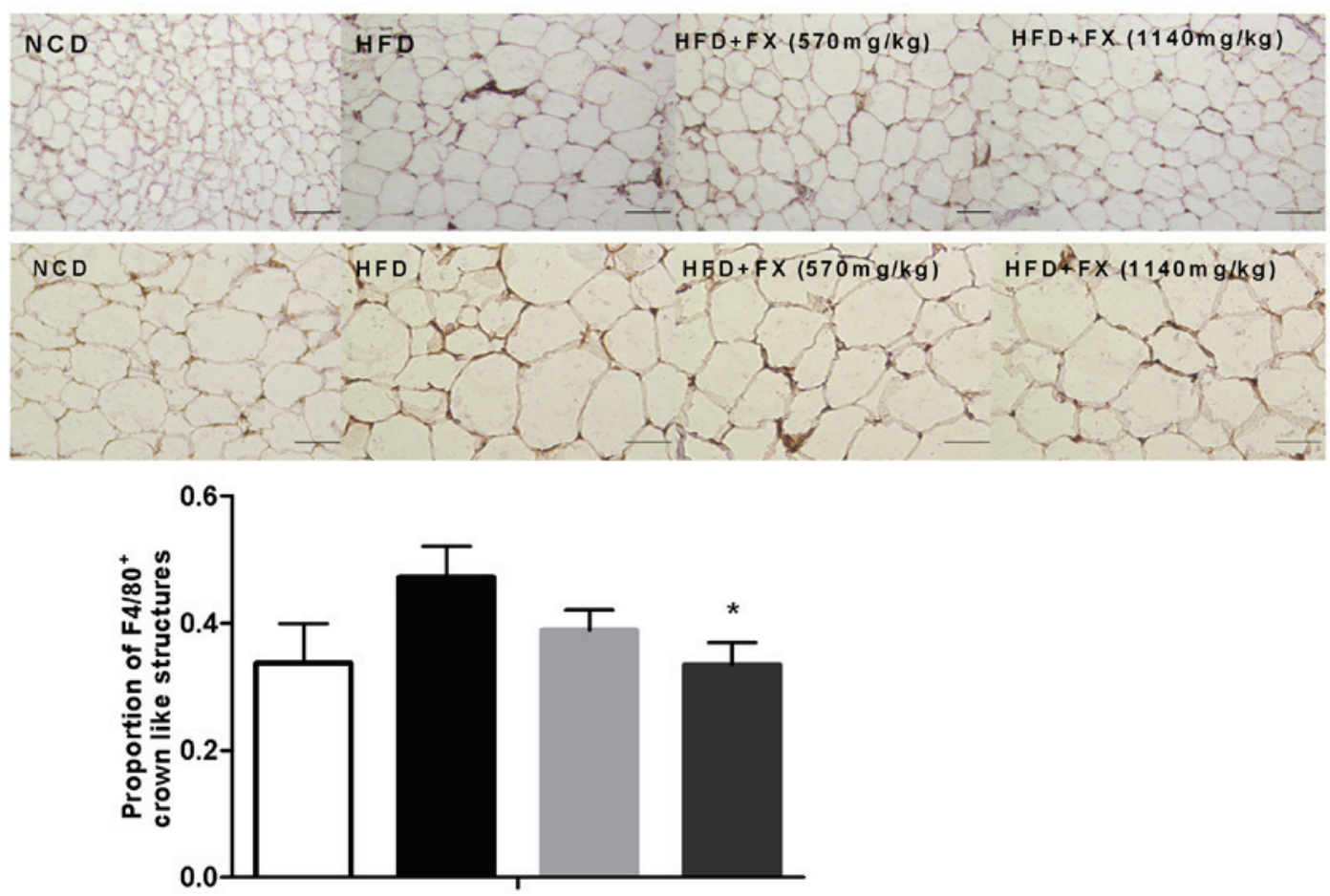

Figure 5. Effect of FX on inflammation in EF. (A) Expression levels of inflammatory genes. Data are presented as the mean \pm standard error of the mean $(\mathrm{n}=10)$. ${ }^{*} \mathrm{P}<0.05$, vs. NCD group; ${ }^{*} \mathrm{P}<0.05$, vs. HFD group. (B) Immunohistochemical staining for F4/80. Scale bar $=200 \mu \mathrm{m}$ (top row) and $100 \mu \mathrm{m}$ (bottom row) Data are presented as the mean \pm standard error of the mean $(\mathrm{n}=5) .{ }^{*} \mathrm{P}<0.05$, vs. HFD group. FX, Fructus xanthii; NCD, normal chow diet; HFD, high fat diet.

were reversed by FX treatment at low and high doses $(\mathrm{P}<0.05)$ as shown in Fig. 1, suggesting a decrease in the release of FFA from the adipose tissues.

$F X$ increases adipocyte size in the EF. Metabolic improvements are generally associated with a reduction in average fat mass and cell size (24). In the present study, histological examination of EF revealed that the average adipocyte size in the HFD group was markedly increased relative to that of the NCD adipocytes, the number of adipocytes in the HFD group was lower than that in the NCD group, and a high dose of FX increased adipocyte number (Fig. 2A and B). Quantitative assessment of adipocyte size distribution clearly demonstrated this shift toward smaller size adipocytes areas in the high dose FX group (Fig. 2C). The AUC of the adipocyte size showed that FX treatment at a high dose increased the AUC of 1,000-25,000 $\mu \mathrm{m}^{2}$, and decreased the AUC of 25,000-50,000 $\mu \mathrm{m}^{2}$ and $>50,000 \mu \mathrm{m}^{2}$ (P>0.05; Fig. 2D).
Effect of FX on lipid metabolism in the EF. The increased release of FFA from adipose tissue has long been linked to lipogenesis and steatosis in the liver (25). In the EF, a HFD increased the mRNA expression levels of carbohydrateresponsive element-binding protein (ChREBP) and SCD1 $(\mathrm{P}<0.05)$, and decreased the levels of SREBP 1c, FAS and acetyl-CoA carboxylase $(\mathrm{ACC} 1 ; \mathrm{P}<0.05)$, which were essentially reversed by FX. Furthermore, FX at a high dose increased the gene expression level of SCD1 significantly $(\mathrm{P}<0.05)$, as shown in Fig. 3. However, treatment with FX had no effect on the expression of fatty acid oxidation-associated genes (carnitine palmitoyltransferase 1, acyl-CoA oxidase and acyl-CoA oxidase 1) or the transcription factor regulating their activities, PPAR $\alpha$. These results demonstrated a role of FX in promoting lipogenesis in EF.

FX switches TG hydrolysis to TG storage. The expression of the TG synthesis-associated genes, GPAT and DPAT, in the 
HFD group were significantly lower, compared with those in the NCD group $(\mathrm{P}<0.05)$. However, a high dose of FX supplementation significantly increased the mRNA expression of DPAT $(\mathrm{P}<0.05)$. No differences were observed in the level of AGPAT among the groups, as shown in Fig. 4A. The mRNA levels of TG lipolysis-associated genes (ATGL and HSL) treated by FX did not differ from the levels in the HFD group, however,he level of ATGL in the HFD group was lower than that in the NCD group $(\mathrm{P}<0.05)$, as shown in Fig. 4B.

In addition, the expression of perilipin, involved in the lipolytic process, was significantly increased $(\mathrm{P}<0.05)$, whereas, FSP-27, another lipid droplet-associated protein, was not modified by FX (Fig. 4C). Fatty acid uptake and transport are important functions for adipose fat storage, and the fatty acid import and transport-associated genes (LPL and CD36) were marginally affected by HFD and NCD. High dose-FX treatment elevated the expression of LPL significantly $(\mathrm{P}<0.05$; Fig. 4D).

$F X$ decreases inflammation in EF. Compared with the NCD group, genes of inflammatory factors, including MCP1 and CD68, were upregulated in the HFD group, whereas the expression of TNF- $\alpha$ was decreased. FX decreased the expression of MCP1 in a dose-dependent manner $(\mathrm{P}<0.05)$, and increased the expression of TNF- $\alpha$ significantly $(\mathrm{P}<0.05)$, as shown in Fig. 5A.

Macrophages are stained brown. In the NCD group, F4/80+ staining were uniformly small, dispersed, and rarely seen in aggregates. The fraction of $\mathrm{F} 4 / 80^{+}$staining was increased and in formed aggregates in the HFD group. FX treatment at a high dose decreased the degree of $\mathrm{F} 4 / 80^{+}$staining, as shown in Fig. 5B. Immunohistochemical analysis showed the total number of $\mathrm{F} 4 / 80^{+}$crown-like structures were higher in the HFD group, compared with the NCD group, although without statistical significance. FX treatment at a high dose suppressed the number of $\mathrm{F} 4 / 80^{+}$crown-like structures $(\mathrm{P}<0.05)$, as shown in Fig. 5B.

\section{Discussion}

Our previous study demonstrated that FX decreases fatty acid synthesis and lipid accumulation in the liver, thus attenuating hepatic steatosis in HFD rats via inhibiting lipogenesis and promoting lipolysis mechanisms, which are associated with the inhibition of ChREBP and SREBP 1c, and the induction of PPAR $\alpha$. This suggested the potential application of FX in treating fatty liver diseases (19). In the present study, it was demonstrated that FX increased the synthesis of fatty acid and TG, thus decreasing the circulating FFA levels, suggesting that FX is involved in addressing the abnormality of FFA in the circulation, which is executed by promoting the storage of the excess fat, rather than the elimination of added fat.

Significant evidence has indicated the ectopic deposition of adipose tissue with respect to its enhanced risk for metabolic complications (21). Adipose tissue stores TG in a positive energy balance condition. Disorder in adipose fat storage function may cause excess fatty acid influx into the liver, leading to steatosis (26). Adipose metabolism acts to buffer nutrient excess in promoting lipid storage with increased adipogenesis and lipogenesis (27). The quantity of TG accumulation in adipose tissue results from the balance of several metabolic pathways, which take place in the tissue, including DNL, fatty acid uptake from circulating TG and lipid mobilization (28).

In the present study, HFD induced hepatic steatosis and high levels of circulating FFA (Fig. 1). Elevated circulating FFA concentration is associated with the accumulation of TG in the liver. Ectopic lipid deposition in the liver (fatty liver) reflects a diminished capacity of adipocytes to store fatty acids, either due to impaired lipogenesis or a diminished capacity for hypertrophy or proliferation (29).

Evidence suggests that one factor, which links excess caloric intake and a positive energy balance with insulin resistance is the inability to appropriately expand the adipose tissue (22). To examine whether the EF is associated with the amelioration of HFD-induced metabolic dysfunction, the present study examined morphological changes, and the sizes and numbers of adipocytes in the EF. HFD-induced fat accumulation in EF was significantly ameliorated, and adipocyte size was markedly reduced in the FX-treated group, in which the distribution of adipocyte size was shifted to smaller sized adipocytes (Fig. 2), showing that FX reduces the burden of HFD, predominantly due to appropriate extended EF, thus reducing fatty acid release from the EF overflux into the liver, and slowed the development of fatty liver.

No significant difference in EF weight was observed between the HFD and FX-treated groups (data not shown). It has become evident that, in addition to absolute fat quantity, qualitative aspects of adipose tissue function and cellular composition have an important effect on the systemic metabolic phenotype (22). Therefore, determined RT-qPCR was used to determine the expression levels of relevant genes involved in lipogenic transcription factors, fatty acid synthesis and esterification, oxidation, TG synthesis enzymes.

DNL in white adipose tissue is considered to occur to a similar extent in the liver (30). In the EF, following HFD, the levels of ChREBP and SCD1 were increased significantly, and FX normalized the high expression level of ChREBP induced by HFD; with the effect of FX at two doses on the level of SCD1 showed the opposite. However, the expression of SREBP 1c was reduced by HFD, together with its target enzymes, FAS and ACC1. FX treatment elevated the expression levels of the three genes significantly, suggesting that, during HFD, lipogenesis of adipose tissue is disordered and causes liver ectopoic lipid deposition (19), and that FX treatment increased fatty acid synthesis, which are critical substrates for the biosynthesis of TG (Fig. 3). These data demonstrated that HFD disturbed the major lipid metabolic pathways in adipose tissue, and FX had regulatory effects suitable to the local situation. Above all, a concomitant increase in the rate of fatty acid synthesis compensated for the increase in fatty acid utilization in EF, preventing an increase in circulating FFA concentrations.

On examination of the genes involved in catalyzing the steps of TG synthesis, the effect of FX on DGAT was determined, which converts diacylglycerol to TG. However, FX had no effect on the expression levels of GPAT or AGPAT. The expression levels of ATGL and HSL, which are two major TG hydrolases responsible for adipose lipolysis, were not significantly affected by FX. Furthermore, the expression of LPL following treatment with FX was increased, compared with that of the HFD group; this indicated a clear effect of 
FX on fatty acid uptake from the circulating TG (Fig. 4). These above data showed that FX transported more fatty acids from the circulation, did not increase the hydrolysis of TG in adipose tissue, and marginally increased the lipogenesis of TG. Therefore, adipose tissue in the FX-treated group buffered HFD-induced TG overproduction, which attenuated liver lipid deposition.

Within a certain range, adipose tissue extension is more conducive to increased fat storage. In addition to this, the number of necrotic adipocytes positively correlates with average adipocyte size in obese mice and other mouse models of adipocyte hypertrophy (31). The death of hypertrophic adipocytes facilitates the infiltration of macrophages, which further perpetuates adipose inflammation and insulin resistance (32). In the present study, high levels of MCP1 and low levels of TNF- $\alpha$ were observed in the HFD group, and despite the controversial results, treatment with FX was shown to attenuate the HFD-induced inflammatory changes. Macrophage infiltration in the EF of the HFD group was marked, indicated by $\mathrm{F} 4 / 80^{+}$staining in brown, however, treatment with FX decreased the staining to differing degrees (Fig. 5). These results suggested that FX limited the inflammatory infiltration, inhibited the vicious cycle of increased inflammation and dysfunction, and maintained the function of EF.

In conclusion, adipose lipid homeostasis is basically dependent on two major functions: TG/fatty acid uptake and lipolysis/fatty acid release (33). FX supplementation reversed NAFLD in association with a reduction in the number of circulating FFA, conveying more FFA from the circulation, and partially increasing the expression levels of the fatty acid and TG synthesis-associated genes in the EF. These data suggest that FX ameliorated HFD-induced lipid dyshomeostasis at the adipose tissue-liver axis.

\section{Acknowledgements}

This study was supported by the Xiamen Science and Technology Bureau (Xiamen Research Platform for Systems Biology of Metabolic Disease; grant no. 3502Z20100001); the National Natural Science Foundation to Dr Shuyu Yang (grant no. 30973912), Dr Xuejun Li (grant no. 81073113), and Dr Suhuan Liu (grant no. 81270901); and the education scientific research project of young teachers in Fujian Province (grant no. JA15306)

\section{References}

1. Handschin C and Spiegelman BM: The role of exercise and PGC1 alpha in inflammation and chronic disease. Nature 454: 463-469, 2008.

2. Duval C, Thissen U, Keshtkar S, Accart B, Stienstra R, Boekschoten MV, Roskams T, Kersten S and Müller M: Adipose tissue dysfunction signals progression of hepatic steatosis towards nonalcoholic steatohepatitis in $\mathrm{C} 57 \mathrm{Bl} / 6$ mice. Diabetes 59: 3181-3191, 2010.

3. Postic C and Girard J: The role of the lipogenic pathway in the development of hepatic steatosis. Diabetes Metab 34 (6 Pt 2): 643-648, 2008.

4. Seo JB, Moo HM, Kim WS, Lee YS, Jeong HW, Yoo EJ, Ham J, Kang H, Park MG, Steffensen KR, et al: Activated liver X receptors stimulate adipocyte differentiation through induction of peroxisome proliferator-activated receptor gamma expression. Mol Cell Biol 24: 3430-3444, 2004.
5. Marcelin G and Chua S Jr: Contributions of adipocyte lipid metabolism to body fat content and implications for the treatment of obesity. Curr Opin Pharmacol 10: 588-593, 2010.

6. Takeuchi K and Reue K: Biochemistry, physiology and genetics of GPAT, AGPAT and lipin enzymes in triglyceride synthesis. Am J Physiol Endocrinol Metab 296: E1195-E1209, 2009.

7. Gimeno RE and Cao J: Thematic review series: Glycerolipids. Mammalian glycerol-3-phosphate acyltransferases: New genes for an old activity. J Lipid Res 49: 2079-2088, 2008.

8. Shindou H, Hishikawa D, Harayama T, Yuki K and Shimizu T: Recent progress on acyl-CoA: Lysophospholipid acyltransferase research. J Lipid Res 50 (Suppl): S46-S51, 2009.

9. Yen CL, Stone SJ, Koliwad S, Harris C and Farese RV Jr: Thematic review series: Glycerolipids. DGAT enzymes and triacylglycerol biosynthesis. J Lipid Res 49: 2283-2301, 2008.

10. Gong J, Sun Z, Wu L, Xu W, Schieber N, Xu D, Shui G, Yang H, Parton RG and Li P: Fsp27 promotes lipid droplet growth by lipid exchange and transfer at lipid droplet contact sites. J Cell Biol 195: 953-963, 2011.

11. Rodriguez-Cuenca S, Carobbio S, Velagapudi VR, Barbarroja N, Moreno-Navarrete JM, Tinahones FJ, Fernandez-Real JM, Orešic M and Vidal-Puig A: Peroxisome proliferator-activated receptor $\gamma$-dependent regulation of lipolytic nodes and metabolic flexibility. Mol Cell Biol 32: 1555-1565, 2012.

12. Wellen KE and Hotamisligil GS: Obesity-induced inflammatory changes in adipose tissue. J Clin Invest 112: 1785-1788, 2003.

13. Sartipy P and Loskutoff DJ: Monocyte chemoattractant protein 1 in obesity and insulin resistance. Proc Natl Acad Sci USA 100: 7265-7270, 2003.

14. Kopelman PG: Obesity as a medical problem. Nature 404: 635-643, 2000.

15. Wanless LR and Lentz JS: Fatty liver hepatitis and obesity: An autopsy study with analysis of risk factors. Hepatology 12: 1106-1110, 1990.

16. Ma P and Li H: The research development of Fructus xanthii. Chinese Herbal Medicine 30: 634-636, 1999.

17. Huang MH, Wang BS, Chiu CS, Amagaya S, Hsieh WT, Huang SS, Shie PH and Huang GJ: Antioxidant, antinociceptive and anti-inflammatory activities of Xanthii Fructus extract. J Ethnopharmacol 135: 545-552, 2011.

18. Song MY, Kim EK, Lee HJ, Park JW, Ryu DG, Kwon KB and Park BH: Fructus Xanthii extract protects against cytokine-induced damage in pancreatic beta-cells through suppression of NF-kappaB activation. Int J Mol Med 23: 547-553, 2009.

19. Li XM, Li ZP, Xue M, Ou ZM, Liu M, Yang M, Liu S, Yang S and $\mathrm{Li}$ X: Fructus Xanthii attenuates hepatic steatosis in rats fed on high-fat diet. Plos one 8: e61499, 2013.

20. Kato K, Takamura T, Takeshita Y, Ryu Y, Misu H, Ota T, Tokuyama K, Nagasaka S, Matsuhisa M, Matsui O and Kaneko S: Ectopic fat accumulation and distant organ-specific insulin resistance in Japanese people with nonalcoholic fatty liver disease. PLoS One 9: e92170, 2014.

21. Lument CN and Saltiel AR: Inflammatory links between obesity and metabolic disease. J Clin Invest 121: 2111-2117, 2011.

22. Kim JY, van de Wall E, Laplante M, Azzara A, Trujillo ME, Hofmann SM, Schraw T, Durand JL, Li H, Li G, et al: Obesity-associated improvements in metabolic profile through expansion of adipose tissue. J Clin Invest 117: 2621-2637, 2007.

23. Livak KJ and Schmittgen TD: Analysis of relative gene expression data using real-time quantitative PCR and the 2[-Delta Delta C(T)] Method. Methods 25: 402-408, 2001.

24. Ginsberg HN, Zhang YL and Hernandez-Ono A: Metabolic syndrome: Focus on dyslipidemia. Obesity (Silver Spring) 14 (Suppl 1): S41-S49, 2006.

25. Sun X, Tang Y, Tan X, Li Q, Zhong W, Sun X, Jia W, McClain CJ and Zhou Z: Activation of peroxisome proliferator-activated receptor- $\gamma$ by rosiglitazone improves lipid homeostasis at the adipose tissue-liver axis in ethanol-fed mice. Am J Physiol Gastrointest Liver Physiol 302: G548-G557, 2012.

26. Khan T, Muise ES, Iyengar P, Wang ZV, Chandalia M, Abate N, Zhang BB, Bonaldo P, Chua S and Scherer PE: Metabolic dysregulation and adipose tissue fibrosis: Role of collage VI. Mol Cell Biol 29: 1575-1591, 2009.

27. Hofbauer KG: Molecular patheays to obesity. Int J Obes Relat Metab Disord 26 (Suppl 2): S18-S27, 2002. 
28. Alberdi G, Rodríguez V, Miranda J, Macarulla M, Arias N Andrés-Lacueva $\mathrm{C}$ and Portillo MP: Changes in white adipose tissue metabolism induced by resveratrol in rats. Nutr Metab (Lond) 8: 29, 2011.

29. Wang M, Grayburn P, Chen S, Ravazzola M, Orci L and Unger RH: Adipogenic capacity and the susceptibility to type 2 diabetes and metabolic syndrome. Proc Natl Acad Sci USA 105: 6139-6144, 2008.

30. Letexier D, Pinteur C,Large V,Fréring V and Beylot M: Comparison of the expression and activity of the lipogenic pathway in human and rat adipose tissue. J Lipid Res 44: 2127-2134, 2003.
31. Strissel KJ, Stancheva Z, Miyoshi H, Perfield JW II, DeFuria J, Jlick Z, Greenberg AS and Obin MS: Adipocyte death, adipose tissue remodeling and obesity complications. Diabetes 56: 2910-2918, 2007.

32. Zhang HR and Zhang CH: Adipose 'talk' to distant organs to regulate insulin sensitivity and vascular function. Obesity (Silver Spring) 18: 2071-2076, 2010.

33. Large V, Peroni O, Letexier D, Ray H and Beylot M: Metabolism of lipids in human white adipocyte. Diabetes Metab 30: 294-309, 2004. 